\title{
Competição entre larvas de Aedes aegypti e Aedes albopictus em laboratório
}

\author{
Competition between Aedes aegypti and Aedes albopictus \\ larvae in the laboratory
}

\author{
Lígia Leandro Nunes Serpa ${ }^{1}$, Iná Kakitani² e Júlio Cesar Voltolini ${ }^{3}$
}

\begin{abstract}
RESUMO
Este estudo teve como objetivo avaliar os efeitos da competição larval intra e interespecífica entre Aedes aegypti eAedes albopictus, sobre sobrevivência de larvas, tempo de desenvolvimento e comprimento de asa. 0 experimento foi realizado em três densidades com 5 proporções das espécies. A sobrevivência de Aedes aegypti apresentou-se superior a de Aedes albopictus em densidade intermediária e inferior em densidade alta. Somente Aedes albopictus teve seu tempo de desenvolvimento afetado. Diferenças encontradas nas comparações das combinações das espécies demonstraram que o comprimento médio de asas de Aedes aegypti, no geral, foi maior que Aedes albopictus. Nas duas espécies, a competição afetou mais o comprimento de asa e a sobrevivência que o tempo de desenvolvimento. Aedes aegypti parece apresentar maior capacidade competitiva em relação a Aedes albopictus em densidade intermediária.
\end{abstract}

Palavras-chaves: Aedes aegypti. Aedes albopictus. Competição intra-específica. Competição interespecífica. Densidade larval.

\begin{abstract}
This study had the aim of evaluating the effects of intra and interspecies larval competition between Aedes aegypti and Aedes albopictus, survival of larvae time taken to develop and wing length. The experiment was carried out with three densities and five proportions of the species. Aedes aegypti survival was greater than Aedes albopictus survival at intermediate density, while it was lower at high density. Only the time taken for Aedes albopictus to develop was affected. The differences found in comparing the species combinations demonstrated that the mean wing length of Aedes aegypti was generally greater than that of Aedes albopictus. For both species, competition had greater effect on wing length and survival than on the time taken to develop. Aedes aegypti seems to present better competitive capacity than does Aedes albopictus, at intermediate density.
\end{abstract}

Key-words: Aedes aegypti. Aedes albopictus. Intraspecies competition. Interspecies competition. Larval density.

Aedes aegypti e Aedes albopictus, mosquitos pertencentes ao subgênero Stegomyia, são espécies que apresentam nichos ecológicos semelhantes. 0 registro de mudanças na distribuição e abundância da espécie residente seja ela Aedes aegypti ou Aedes albopictus, após o estabelecimento da espécie invasora, sugere a possibilidade da ocorrência do deslocamento competitivo entre as mesmas ${ }^{3}$.

A influência da competição intra-específica nessas espécies é reconhecida ${ }^{2}$. Segundo Lord $^{6}$, alta densidade larval intraespecífica e escassa disponibilidade de alimento no criadouro causam efeito negativo sobre os indivíduos, consequientemente afeta o crescimento populacional de mosquitos.
Além dos efeitos da competição intra-específica, experimentos de competição entre larvas dessas espécies, com diferentes populações geográficas, têm sugerido a ocorrência da competição interespecífica. Há décadas, procura-se explicar e predizer mudanças no padrão populacional dessas espécies, seja pelo estudo da ação da interferência química, tipo e/ou escassez de alimento, densidade larval ${ }^{17}$, entre outros fatores. A maior capacidade competitiva foi apontada para Aedes albopictus, em experimentos de laboratório e campo, no uso de dieta natural, folha em decomposição e, principalmente em alta densidade larval ${ }^{15}$. Para Aedes aegypti, a superioridade competitiva foi declarada em experimentos de laboratório, no uso de dieta $\operatorname{artificial}^{713}$.

\footnotetext{
1. Seção Técnica de Pesquisa em Vetores, Superintendência de Controle de Endemias, Taubaté, SP. 2. Faculdade de Saúde Pública, Universidade de São Paulo, São Paulo, SP. 3. Universidade de Taubaté, Taubaté, SP.

Apoio: Fundação de Amparo à Pesquisa do Estado de São Paulo (FAPESP).

Endereço para correspondência: MSc Lígia Leandro Nunes Serpa. Seção Técnica de Pesquisa em Vetores/SUCEN. Praça Coronel Vitoriano 23, Jardim Santa Clara, 12020-020 Taubaté, SP.

Tel: $55123632-7616$

e-mail: ligiaserp@sucen.sp.gov.br

Recebido para publicação em 19/03/2008

Aceito em 27/08/2008
} 
No Brasil, experimento de competição em campo, com Aedes aegypti e Aedes albopictus do Estado do Rio de Janeiro, Braks e cols ${ }^{3}$ verificaram a superioridade competitiva de Aedes albopictus.

No Estado de São Paulo, onde a coexistência de larvas destas espécies em criadouros artificiais de área urbana tem sido registrada com frequiência, ao longo dos anos de 1996 até 2000, observações de campo indicaram predominância de Aedes aegypti em relação a Aedes albopictus ${ }^{9}$. Atualmente, essas espécies coexistem em $80 \%$ das Cidades do Estado de São Paulo. Em Potim, município paulista pertencente à Região do Vale do Paraíba, o estabelecimento desses mosquitos se deu em diferentes décadas, Aedes albopictus em 1986 e Aedes aegypti no ano de 2000. Entretanto, a sobreposição dessas espécies se dá em 13 dos 35 municípios existentes na região, onde ocorre também transmissão dos vírus da dengue, segundo dados da Superintendência de Controle de Endemias da Secretaria de Estado da Saúde de São Paulo. Naquele município, o estudo da variação sazonal de Aedes aegypti e Aedes albopictus revelou maior frequiência de Aedes aegypti em todas as estações do ano tendo sido registrada uma amplitude de 590 a 3.048 indivíduos. JáAedes albopictus foi registrado em épocas do ano de maior abundância para Culicídeos, porém em número reduzido com amplitude de 0 a 484 indivíduos ${ }^{11}$. Diante do exposto, investigar a ocorrência da competição larval nessas espécies, em diferentes densidades, pode fornecer indícios de favorecimento na interação.

Nosso objetivo foi testar, em laboratório, as hipóteses de: maior capacidade competitiva de Aedes aegypti, favorecimento de Aedes albopictus em condições de superpopulação larval, e ocorrência de competição intra-específica para as duas espécies em alta densidade larval. 0 efeito da interação foi avaliado quanto ao tempo de desenvolvimento de larvas de primeiro estádio até pupa, sobrevivência de larvas e tamanho de fêmeas adultas.

\section{MATERIAL E MÉTODOS}

Local de estudo. 0 experimento foi realizado no Laboratório de Pesquisa da Superintendência de Controle de Endemias -

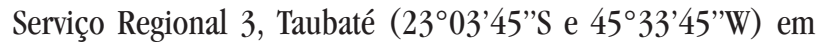
fotoperíodo de $14 \mathrm{~L}: 10 \mathrm{E}$ e temperatura média de $25,5^{\circ} \mathrm{C}$ da água.

Planejamento da amostragem. As larvas utilizadas neste experimento foram de primeira geração de indivíduos de Aedes aegypti coletados no município de Potim (22 48'45"S e $45^{\circ} 18^{\prime} 45^{\prime \prime W}$ ), único infestado por esta espécie no período de estudo e, Aedes albopictus nos municípios de Tremembé

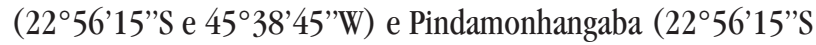
e $45^{\circ} 26$ ' $15^{\prime \prime W}$ ). Estes dois últimos municípios são distantes $10 \mathrm{~km}$ e todos pertencentem à Região do Vale do Paraíba, São Paulo, Brasil.

A competição larval intra e interespecífica foi investigada pelo uso do desenho experimental de substituição em série, poderoso método de avaliação da competição em experimento único ${ }^{8}$. Entretanto, o experimento foi realizado uma única vez, em função do grande número de larvas necessárias, área física requerida e capacidade operacional disponível. Foram utilizadas combinações de Aedes aegypti e Aedes albopictus nas proporções de 10:0 - 7:3 - 5:5 - 3:7 - 0:10, respectivamente. 0 desenvolvimento larval se deu em densidades baixa (360 larvas/litro), intermediária (1.200 larvas/litro) e alta (4.000 larvas/litro) (Tabela 1).

Tabela 1 - Organização do experimento de competição de Aedes aegypti $\boldsymbol{e}$ Aedes albopictus.

\begin{tabular}{|c|c|c|c|}
\hline & Baixa & $\begin{array}{c}\text { Densidade } \\
\text { intermediária }\end{array}$ & Alta \\
\hline & $(360$ & $(1.200$ & $(4.000$ \\
\hline & larvas/litro) & larvas/litro) & larvas/litro) \\
\hline Volume de água & $167 \mathrm{ml}$ & $117 \mathrm{ml}$ & $50 \mathrm{ml}$ \\
\hline \multicolumn{4}{|l|}{ Competição intra-específica } \\
\hline \multicolumn{4}{|l|}{ (Controle) } \\
\hline 100 & 60 & 140 & 200 \\
\hline \multicolumn{4}{|l|}{ Competição interespecífica } \\
\hline $7: 3$ & $42: 18$ & $98: 42$ & $140: 60$ \\
\hline $5: 5$ & $30: 30$ & $70: 70$ & $100: 100$ \\
\hline $3: 7$ & $18: 42$ & $42: 98$ & $60: 140$ \\
\hline \multirow{2}{*}{\multicolumn{4}{|c|}{$\begin{array}{l}\text { Quantidade de recurso alimentar } \\
\text { disponível }(\mathrm{mg} / \mathrm{ml})\end{array}$}} \\
\hline & & & \\
\hline $1^{\circ} \mathrm{dia}(0,1 \mathrm{mg} / \mathrm{ml})$ & 16,7 & 11,7 & 5,0 \\
\hline $3^{0} \mathrm{dia}(0,2 \mathrm{mg} / \mathrm{ml})$ & 33,4 & 23,4 & 10,0 \\
\hline $5^{\circ} \mathrm{dia}(0,3 \mathrm{mg} / \mathrm{ml})$ & 50,1 & 35,1 & 15,0 \\
\hline $7^{0} \mathrm{dia}(0,4 \mathrm{mg} / \mathrm{ml})$ & 66,8 & 46,8 & 20,0 \\
\hline $9^{\circ}$ dia ao final do experimento & 33,4 & 23,4 & 10,0 \\
\hline$(0,2 \mathrm{mg} / \mathrm{ml})$ & & & \\
\hline
\end{tabular}

Competição larval. Sobrevivência e tempo de desenvolvimento: a competição intra-específica serviu também como controle da interespecífica. As larvas foram coletadas por meio de pipeta de vidro, com auxílio de foco luminoso, e mantidas em copos plásticos descartáveis, na proporção de 1 larva/2ml de água destilada. 0 tempo de espera não ultrapassou 18 horas de vida até que se montasse o experimento propriamente dito.

Cada recipiente de criação foi constituído de cuba de vidro medindo $10,5 \mathrm{~cm}$ de diâmetro e 4,5cm de altura, com capacidade máxima de $300 \mathrm{ml}$.

A quantidade de alimento foi calculada por ml de água, fator limitante no desenvolvimento quando a densidade larval excede 1 larva $/ 0,75 \mathrm{ml}$ de água ${ }^{10}$. Dessa forma a quantidade de alimento fornecida, ração para peixes, foi diferente entre as três densidades e escassa em densidade alta (Tabela 1). Também em dias alternados, coincidindo com o fornecimento do alimento, $2 / 3$ do volume de água foi substituído por água destilada. Tal procedimento se deu na intenção de reduzir a poluição da água $^{2}$. As pupas foram diariamente removidas de cada unidade experimental. Em seguida, transferidas individualmente para copos plásticos telados de $3,5 \mathrm{~cm}$ de diâmetro e $3,5 \mathrm{~cm}$ de altura. Neste foi acrescido $10 \mathrm{ml}$ de água destilada. A morte dos adultos se deu pelo uso de acetato de etila, após um período máximo de 24 horas da sua emergência. Para a análise dos efeitos da competição larval intra e interespecífica, sobre a sobrevivência de larvas, considerou-se o número total de pupas. Nas análises do tempo de desenvolvimento foi considerado, em densidades baixa e intermediária, $50 \%$ do total de larvas, ou seja, a primeira metade 
do total de larvas a atingir o estágio pupal e, 100\% das pupas de densidade alta, uma vez que nesta a mortalidade de Aedes aegypti foi superior a $50 \%$ do total da amostra. Foram feitas comparações nas diferentes densidades e também entre as espécies utilizando-se um teste para proporções independentes ${ }^{15}$. Após, para a correção de Bonferroni utilizamos o valor de alfa corrigido de 0,002 resultante da divisão do número de comparações (21) pelo valor de alfa convencional $(0,05)$.

Comprimento de asa: uma asa de cada fêmea foi dissecada para estimar o tamanho do corpo do mosquito adulto, devido ao pequeno número de fêmeas emergentes no final do experimento.

A medição foi feita com o uso de microscópio binocular do chanfro alular até a margem distal, excluindo as escamas ${ }^{14}$.

Foram comparadas todas as fêmeas adultas emergentes nas três densidades utilizando o teste de Kruskal-Wallis e o teste de Dwass-Steel-Chritchlow-Flingner para as comparações múltiplas $($ alfa $=0,05)$. As análises foram realizadas através do software Statistica 5.0 e Statsdirect 2.3.7 .

\section{RESULTADOS}

Sobrevivência de larvas. Efeito da competição intraespecífica: foi verificado na densidade intermediária e alta. Naquela, observou-se aumento da sobrevivência de Aedes albopictus quando comparada a combinação 5:5, ou seja, na densidade de 1.200 larvas/litro a sobrevivência do controle foi superior $(p=0,00)$. Contrariamente, em densidade alta, a sobrevivência desta mesma espécie apresentou-se diminuída quando comparada às combinações as combinações de 7:3 $(\mathrm{p}=0,00)$ e 3:7 $(\mathrm{p}=0,00)$ apresentando-se semelhante apenas à combinação de mesma proporção das espécies $(\mathrm{p}=0,32)$. Para Aedes aegypti verificou-se diminuição da sobrevivência apenas em densidade alta (4.000larvas/litro) e somente na combinação 3:7 $(\mathrm{p}=0,00)$ (Tabela 2).

Efeito da competição interespecífica: diferenças foram verificadas em densidade intermediária e alta. Na densidade intermediária, para a combinação de igual número das espécies (5:5), Aedes aegypti apresentou maior sobrevivência $(\mathrm{p}=0,00)$. Já em densidade altaAedes albopictus teve maior sobrevivência nas três combinações das espécies. Verificou-se, ainda em densidade alta, sobrevivência aumentada de Aedes aegypti, em comparação ao seu respectivo controle, quando o número de larvas dessa espécie foi o menor da combinação (3:7) (Tabela 2).

Tempo de desenvolvimento. Efeito da competição intraespecifica: as diferenças ocorreram apenas na densidade alta e somente para Aedes albopictus. Essa espécie registrou menor tempo de desenvolvimento no controle do que na combinação de diferentes proporções das espécies (Tabela 3).

Efeito da competição interespecífica: as diferenças foram registradas também apenas em alta densidade, onde Aedes albopictus apresentou menor tempo de desenvolvimento nas três combinações das espécies $(7: 3 ; 5: 5 ; 3: 7)$ (Tabela 3).
Tabela 2 - Sobrevivência intra e interespecífica de larvas de Aedes aegypti $\boldsymbol{e}$ Aedes albopictus até pupa, nas três densidades.

\begin{tabular}{|c|c|c|c|c|c|c|c|c|}
\hline \multicolumn{3}{|c|}{$\begin{array}{l}\text { Densidade baixa } \\
\text { (360 larvas/litro) }\end{array}$} & \multicolumn{4}{|c|}{ Sobreviventes } & \multicolumn{2}{|c|}{$\begin{array}{l}\% \text { sobreviventes } \\
\text { total sobreviventes }\end{array}$} \\
\hline$\%$ & $\mathrm{n}$ aeg & $\mathrm{n}$ alb & $\operatorname{aeg} n^{0}$ & alb $n^{0}$ & aeg \% & alb\% & aeg & alb \\
\hline 100 & 60 & 0 & 58 & 0 & 97 & 0 & 97 & 0 \\
\hline 70 & 42 & 18 & 41 & 17 & 98 & 94 & 71 & 29 \\
\hline 50 & 30 & 30 & 29 & 26 & 97 & 87 & 53 & 47 \\
\hline 30 & 18 & 42 & 17 & 31 & 94 & 74 & 35 & 65 \\
\hline 0 & 0 & 60 & 0 & 51 & 0 & 85 & 0 & 85 \\
\hline \multicolumn{3}{|c|}{$\begin{array}{l}\text { Densidade intermediária } \\
\text { (1.200 larvas/litro) }\end{array}$} & & Sobrev & entes & & \multicolumn{2}{|c|}{$\begin{array}{l}\% \text { sobreviventes } \\
\text { total sobreviventes }\end{array}$} \\
\hline$\%$ & $\mathrm{n}$ aeg & $\mathrm{n}$ alb & $\operatorname{aeg} \mathrm{n}^{0}$ & alb $n^{\underline{0}}$ & aeg \% & alb\% & aeg & alb \\
\hline 100 & 140 & 0 & 134 & 0 & 96 & 0 & 96 & 0 \\
\hline 70 & 98 & 42 & 98 & 42 & 100 & 100 & 70 & 30 \\
\hline 50 & 70 & 70 & 70 & 51 & 100 & 73 & 58 & 42 \\
\hline 30 & 42 & 98 & 42 & 84 & 100 & 86 & 33 & 67 \\
\hline 0 & 0 & 140 & 0 & 133 & 0 & 95 & 0 & 68 \\
\hline \multicolumn{3}{|c|}{$\begin{array}{l}\text { Densidade alta } \\
\text { (4000 larvas/litro) }\end{array}$} & & Sobrev & ntes & & \multicolumn{2}{|c|}{$\begin{array}{l}\text { \% sobreviventes } \\
\text { total sobreviventes }\end{array}$} \\
\hline$\%$ & $\mathrm{n}$ aeg & $\mathrm{n}$ alb & $\overline{a e g} n^{0}$ & alb $n^{0}$ & $\operatorname{aeg} \%$ & alb\% & aeg & alb \\
\hline 100 & 200 & 0 & 41 & 0 & 21 & 0 & 20,5 & 0 \\
\hline 70 & 140 & 60 & 37 & 53 & 26 & 88 & 41 & 59 \\
\hline 50 & 100 & 100 & 21 & 82 & 21 & 82 & 20 & 80 \\
\hline 30 & 60 & 140 & 25 & 132 & 42 & 94 & 16 & 84 \\
\hline 0 & 0 & 200 & 0 & 153 & 0 & 77 & 0 & 38,5 \\
\hline
\end{tabular}

aeg: Aedes aegypti, alb: Aedes albopictus, N: tamanho da amostra

Tabela 3 - Tempo de desenvolvimento intra-específico de larvas de Aedes albopictus e interespecífico de larvas de Aedes aegypti $\boldsymbol{e}$ Aedes albopictus, ambos até pupa, nas três densidades. Oprimeiro número da combinação corresponde ao Aedes aegypti $\boldsymbol{e}$ o segundo, Aedes albopictus.

\begin{tabular}{|c|c|c|c|c|c|c|}
\hline \multirow{3}{*}{$\begin{array}{l}\text { Proporção } \\
\text { das } \\
\text { espécies }\end{array}$} & \multicolumn{6}{|c|}{ Tempo de desenvolvimento (dias) } \\
\hline & \multicolumn{2}{|c|}{ densidade baixa } & \multicolumn{2}{|c|}{ densidade intermediária } & \multicolumn{2}{|c|}{ densidade alta } \\
\hline & aeg & alb & aeg & alb & aeg & alb \\
\hline 100 & 10 & - & 16 & - & 40 & - \\
\hline $7: 3$ & 10 & 9 & 16 & 15 & 47 & 42 \\
\hline $5: 5$ & 9 & 8 & 15 & 14 & 38 & 37 \\
\hline $3: 7$ & 8 & 9 & 15 & 15 & 44 & 40 \\
\hline 0 & - & 10 & - & 18 & - & 27 \\
\hline
\end{tabular}

aeg: Aedes aegypti, alb: Aedes albopictus

Comprimento da asa de fêmeas. 0 total de fêmeas emergentes teve uma asa medida. Como o número de indivíduos sobreviventes variou de acordo com o tratamento, foi medido no final do experimento entre 7 e 28, 13 e 63 e, 6 e 12 indivíduos de Aedes aegypti e entre 12 e 30, 24 e 71 e, 33 e 73 indivíduos de Aedes albopictus das densidades baixa, intermediária e alta, respectivamente. 0 comprimento médio de asa entre cada densidade e nas duas espécies, foi diferente (teste de Kruskal-Wallis, $\mathrm{H}=605,73$; $\left.\mathrm{n}^{\mathrm{o}}=754 ; \mathrm{gl}=23 ; \mathrm{P}=0,001\right)$. Após, segundo o teste de comparações múltiplas, identificamos diferenças entre as espécies na competição interespecífica. 0 comprimento médio da asa nas duas espécies diminuiu à medida que as densidades aumentaram (Figura 1).

Efeito da competição intra-específica: na comparação dos controles com suas respectivas culturas mistas verificou-se semelhança nas três densidades analisadas separadamente, tanto para Aedes aegypti quanto para Aedes albopictus. 


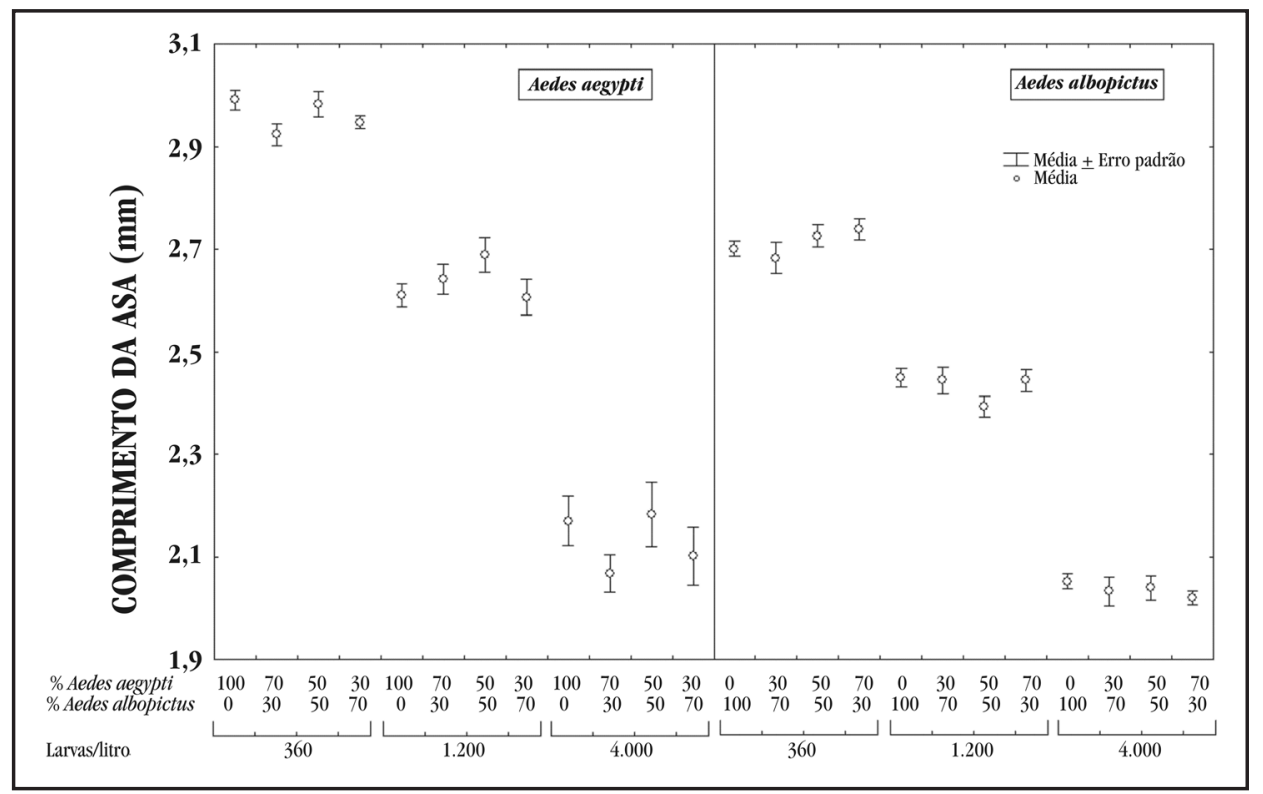

Figura 1 - Comprimento médio de asa de fêmeas de Aedes aegypti $\boldsymbol{e}$ Aedes albopictus de culturas puras e mistas em três densidades.

Efeito da competição interespecífica: nas comparações das espécies de culturas mistas de cada densidade separadamente, foram verificadas diferenças significantes. Aedes aegypti só não apresentou maior comprimento médio de asa que Aedes albopictus nas combinações 3:7 X 7:3 das densidades baixa $(\mathrm{p}=0,07)$ e intermediária $(\mathrm{p}=0,32)$ e 3:7X3:7 de densidade intermediária $(\mathrm{p}=0,12)$. Já em densidade alta, apresentaramse semelhantes nas três combinações das espécies (Teste de comparações múltiplas de DSCF).

As asas de Aedes aegypti dos controles foram sempre maiores que as dos controles da outra espécie, em comparações feitas dentro de cada uma das três densidades. (densidades baixa e intermediária $p=0,00$, densidade alta $p=0,02)$. Embora com o aumento da densidade as asas dos indivíduos nos controles tenham diminuído em ambas as espécies observou-se que Aedes aegypti do controle, de densidade intermediária, apresentou semelhança com Aedes albopictus do controle de densidade baixa $(p=0,63)$. Esse padrão de semelhança pôde ser constatado na maioria das comparações das combinações das espécies dessas mesmas densidades (Teste de comparações múltiplas de DSCF).

\section{DISCUSSÃO}

Sobrevivência de larvas. A sobrevivência larval intraespecífica de ambas as espécies não foi afetada em densidade baixa e intermediária. Entretanto, seus efeitos negativos foram evidenciados, para Aedes aegypti e Aedes albopictus, na maior densidade estudada (4.000larvas/litro). 0 efeito redutor da sobrevivência na competição intra-específica das duas espécies, observado em densidade alta, foi anteriormente indicado por diferentes autores ${ }^{16}$. No presente estudo, foi verificada maior sobrevivência de Aedes albopictus em relação a Aedes aegypti de densidade alta demonstrando a maior resistência daquela espécie à superpopulação larval intra-específica, conforme verificado por Barrera ${ }^{1}$. Bèdhomme e cols², no estudo da poluição da água como componente da competição intra-específica em larvas de Aedes aegypti, verificaram que parte dos efeitos negativos são provocados pela excreção de substâncias larvais. Segundo os mesmos autores, a presença destas substâncias, aliadas a redução de alimento, ou outro processo de modificação do ambiente, são diretamente proporcionais a densidade larval do criadouro. No presente estudo, embora se tenha realizada troca de $2 / 3$ da água anteriormente ao fornecimento de alimento, é possível que, em densidade alta, as condições ambientais tenham sido semelhantes àquelas referidas por tais autores. 0 recurso alimentar disponível pode também ter afetado sensivelmente o estado nutricional das larvas deAedes aegypti, conseqüentemente, sua sobrevivência uma vez que a densidade de larvas, conforme mencionado por Peters e cols ${ }^{10}$ no estudo da padronização de técnica de criação de Aedes aegypti para avaliação do processo competitivo, foi superior a 0,75 larvas $/ \mathrm{ml}$.

0 efeito da competição interespecífica pôde ser expresso por um crescimento populacional positivo verificado para ambas as espécies em densidade intermediária e alta. Naquela, a sobrevivência de Aedes aegypti foi superior a Aedes albopictus, quando a proporção das espécies era a mesma (5:5). Moore e Fischer ${ }^{7}$, semelhantemente, verificaram os efeitos negativos da interação de Aedes aegypti sobre a sobrevivência larval de Aedes albopictus. Já em alta densidade, a maior tolerância de Aedes albopictus em relação à Aedes aegypti, verificada no presente estudo, foi também registrada por Juliano e cols ${ }^{5}$ em experimento de competição em campo, realizado no final da estação chuvosa, quando as densidades larvais foram as maiores. Tais autores mencionaram que 0 aparente impacto da competição mostrou-se variar sazonalmente e estava associado com o número de imaturos/vaso. Apesar da menor sobrevivência de 
Aedes aegypti comparada a de Aedes albopictus em densidade alta, foi observada, na combinação 3:7, o dobro de sobrevivência da primeira espécie, quando comparada ao seu controle, o que sugere maior efeito da competição intra-específica. Assim, a atratividade de fêmeas grávidas de Aedes aegypti por água contendo Aedes albopictus, conforme verificado por Sucharit e cols ${ }^{12}$, possivelmente ocorra devido aos menores efeitos da competição interespecífica quando comparado a intra-específica de alta densidade. Bédhomme e cols ${ }^{2}$ observaram efeitos negativos intra-específicos de Aedes aegypti sobre o tempo de desenvolvimento, longevidade de adultos e tamanho/peso do corpo de indivíduos que se desenvolveram em água onde larvas co-específicas já haviam se desenvolvido.

Embora Aedes albopictus tenha apresentado maior tolerância à superpopulação larval, notou-se efeito benéfico da interação em densidade alta. Nesta situação, a sobrevivência de ambas as espécies foi significativamente aumentada, sendo a de Aedes aegypti especificamente na combinação 3:7 e Aedes albopictus nas três combinações das espécies. Tal comportamento sugere que os efeitos da competição intra-específica sobre a sobrevivência, em ambas espécies, sejam mais intensos que o interespecífico.

Certamente, o estudo do efeito de água onde larvas coespecíficas e hetero-específicas tenham se desenvolvido, no que se refere a oviposição de fêmeas grávidas, possa contribuir no melhor entendimento da relação ecológica dessas espécies.

Tempo de desenvolvimento. No que se refere ao efeito da competição intra-específica, as análises revelaram diferença no tempo de desenvolvimento de Aedes albopictus em densidade alta. Esta espécie apresentou menor tempo de desenvolvimento no controle, quando comparado às culturas mistas das três combinações das espécies (Tabela 3). 0 efeito dependente de densidade sobre o tempo de desenvolvimento parece ser mediado parcialmente pela disponibilidade de alimento, uma vez que outros processos, possivelmente, atuem como a interferência física ou química ${ }^{6}$.

Quando as espécies foram criadas juntas, nas três densidades, houve semelhança no tempo de desenvolvimento de Aedes aegypti com seus respectivos controles. Já para Aedes albopictus verificou-se diferença no tempo de desenvolvimento e somente em densidade alta. Neste caso, houve aumento em culturas mistas quando comparado ao respectivo controle (Tabela 3). Larvas de Aedes aegypti podem provocar efeito quantitativo sobre larvas de Aedes albopictus, aumentando o tempo de desenvolvimento desta espécie, conforme verificado semelhantemente por Sucharit e cols ${ }^{13}$. Moore e Fischer ${ }^{7}$ observaram redução marcante do tempo de desenvolvimento de ambas as espécies, quando em densidade larval alta a porcentagem de Aedes aegypti diminuiu.

Comprimento de asa de fêmeas. Embora Aedes aegypti e Aedes albopictus do controle tenham apresentado semelhança, quando comparado as três densidades de culturas mistas, separadamente, verificou-se que com o aumento da densidade, as asas diminuíram. Tais dados demonstraram variação intraespecífica para ambas as espécies. Observações semelhantes, em Aedes albopictus, foram feitas por Braks e cols ${ }^{3}$. Entretanto, para Aedes aegypti, esses mesmos autores não registraram quaisquer diferenças significantes. Lord ${ }^{6}$ verificou, em Aedes albopictus, que 0 aumento da densidade larval e a redução na disponibilidade de alimento, ou ambos, resultou em adultos sobreviventes menores. No presente estudo é possível mencionar a ocorrência de sua diminuição com o aumento da densidade uma vez que 0 alimento foi fornecido em função do volume de água ${ }^{10}$. Assim, provavelmente a redução no comprimento da asa tenha sido influenciada pela quantidade de alimento disponível.

A competição interespecífica afetou o comprimento médio da asa das fêmeas das duas espécies estudadas, diminuindo seu tamanho conforme as densidades aumentaram (Figura 1).

Observou-se que Aedes aegypti apresentou maior comprimento médio de asa quando comparado a Aedes albopictus dentro das mesmas densidades, baixa e intermediária. Porém, em densidade alta, onde Aedes aegypti apresentou alta mortalidade, as espécies apresentaram-se semelhantes. Sendo assim, deve-se considerar a possibilidade de tal resultado ter ocorrido devido ao número pequeno de sobreviventes. Quando comparamos o comprimento médio da asa de Aedes albopictus do controle de densidade baixa com Aedes aegypti do controle de densidade intermediária contata-se que as mesmas possuem comprimentos semelhantes.

Embora não se tenha avaliado o potencial reprodutivo das fêmeas, os resultados mostraram algumas vantagens competitivas de Aedes aegypti. Isto porque, essa espécie pôde completar o desenvolvimento larval em densidade intermediária com maior sucesso que Aedes albopictus. Além disso, apresentou no controle e em algumas combinações das espécies de densidades baixa e intermediária, comprimento médio de asa das fêmeas maior do que da outra espécie. Tais resultados são de grande interesse epidemiológico uma vez que, segundo Gleiser e cols ${ }^{4}$, fatores bionômicos de Culicidae como: fecundidade, dispersão espacial e frequiência de picadas no hospedeiro podem ser influenciados pelo tamanho do mosquito adulto. Seria interessante verificar em condições naturais se esse padrão de comprimento médio de asa das fêmeas das duas espécies se mantém, além de avaliar o potencial reprodutivo das fêmeas emergentes em situação de coexistência no criadouro.

Conclui-se que as diferenças detectadas na sobrevivência, tempo de desenvolvimento e tamanho de asa sugerem indícios de competição intra e interespecífica em larvas de Aedes aegypti e Aedes albopictus. 0 comprimento de asa foi mais afetado pela competição do que a sobrevivência e tempo de desenvolvimento. Aedes aegypti parece apresentar maior capacidade competitiva em densidade intermediária. Diante do exposto, investigar a competição larval sazonal de Aedes aegypti e Aedes albopictus em campo poderá esclarecer o padrão comportamental dessas espécies nesta região.

\section{REFERÊNCIAS}

1. Barrera R. Competition and resistance to starvation in larvae of containerinhabiting Aedes mosquitoes. Ecological Entomology 21:117-127, 1996.

2. Bédhomme S, Agnew P, Sidobre C, Michalakis Y. Pollution by conspecifics as a component of intraspecific competition among Aedes aegypti larvae. Ecological Entomology 30:1-7, 2005. 
3. Braks MAH, Honório NA, Lounibos LP, Lourenço-de-Oliveira R, Juliano SA. Interespecific competition between two invasive species of container mosquitoes, Aedes aegypti and Aedes albopictus (Diptera: Cullicidae), in Brasil. Annuals of the Entomological Society of America 97:130-139, 2004.

4. Gleiser RM, Urrutia J, Gorla DE. Body size variation of the floodwater mosquito Aedes albifasciatus in Central Argentina. Medical and Veterinary Entomology 14:38-43, 2000.

5. Juliano SA, Lounibos LP, O'Meara GF. A field test for competitive effects of Aedes albopictus on Ae. aegypti in South Florida: differences between sites of coexistence and exclusion? Oecologia 139:583-593, 2004

6. Lord CC. Density dependence in larval Aedes albopictus (Diptera: Culicidae). Journal of Medical Entomology 35:825-829, 1998.

7. Moore CG, Fischer BR. Competition in mosquitoes. Density and species ratio effects on growth, mortality, fecundity, and production of growth retardant. Annals of the Entomological Society of America 62:1325-1331, 1969.

8. Novak MG, Higley LG, Christianssen CA, Rowley WA. Evaluating larval competition between Aedes albopictus and A. triseriatus (Diptera: Culicidae) through replacement series experiments. Environmental Entomology 22: 311-318, 1993.
9. Passos RA, Marques GRAM, Voltolini JC, Condino MLF. Dominância de Aedes aegypti sobre Aedes albopictus no litoral sudeste do Brasil. Revista de Saúde Pública 37:729-734, 2003.

10. Peters TM, Chevone BI, Greenough NC, Callahan RA, Barbosa P. Intraspecific competition in Aedes aegypti (L.) larvae: I. Equipment, techniques and methodology. Mosquito News 29:667-674, 1969.

11. Serpa LLN, Costa KVRM, Kakitani I, Voltolini JC. Variação Sazonal deAedes aegypti eAedes albopictus no município de Potim, São Paulo. Revista de Saúde Pública 40:1101-1105, 2006

12. Sucharit S, Tumrasvin W, Vutikes S, Leemingsawart S. The presence of oviposition attractans of Aedes albopictus larval holding water on Aedes aegypti. Southeast Asian Journal of Tropical Medicine and Public Health 11:417-418, 1980.

13. Sucharit S, Tumrasvin W, Vutikes S, Viraboonchai S. Interactions between larvae of Aedes aegypti and Aedes albopictus in mixed experimental populations. Southeast Asian Journal of Tropical Medicine and Public Health 9:93-97, 1978.

14. Washburn J0, Anderson JR, Mercer DR. Emergence characteristics of Aedes sierrensis (Diptera: Culicidae) from California treeholes with particular reference to parasite loads. Journal of Medical Entomology 26:173-182, 1989.

15. Zar JH. Biostatistical analysis. $4^{a}$ edição, Prentice Hall, Englewood Cliffs, 1999. 